\title{
Inhibitory effect of vitamin D-binding protein-derived macrophage activating factor on DMBA-induced hamster cheek pouch carcinogenesis and its derived carcinoma cell line
}

\author{
YUKIYO TOYOHARA ${ }^{1}$, SUSUMU HASHITANI ${ }^{1}$, HIROMITSU KISHIMOTO ${ }^{1}$, \\ KAZUMA NOGUCHI $^{1}$, NOBUTO YAMAMOTO ${ }^{2}$ and MASAHIRO URADE ${ }^{1}$ \\ ${ }^{1}$ Department of Oral and Maxillofacial Surgery, Hyogo College of Medicine, Hyogo 663-8501, Japan; ${ }^{2}$ Division of Cancer \\ Immunology and Molecular Biology, Socrates Institute for Therapeutic Immunology, Philadelphia, PA 19126-3305, USA
}

Received December 15, 2010; Accepted April 7, 2011

DOI: $10.3892 / \mathrm{ol} .2011 .306$

\begin{abstract}
This study investigated the inhibitory effect of vitamin D-binding protein-derived macrophage-activating factor (GcMAF) on carcinogenesis and tumor growth, using a 9,10-dimethyl-1,2-benzanthracene (DMBA)-induced hamster cheek pouch carcinogenesis model, as well as the cytocidal effect of activated macrophages against HCPC-1, a cell line established from DMBA-induced cheek pouch carcinoma. DMBA application induced squamous cell carcinoma in all 15 hamsters of the control group at approximately 10 weeks, and all 15 hamsters died of tumor burden within 20 weeks. By contrast, 2 out of the 14 hamsters with GcMAF administration did not develop tumors and the remaining 12 hamsters showed a significant delay of tumor development for approximately 3.5 weeks. The growth of tumors formed was significantly suppressed and none of the hamsters died within the 20 weeks during which they were observed. When GcMAF administration was stopped at the 13th week of the experiment in 4 out of the 14 hamsters in the GcMAF-treated group, tumor growth was promoted, but none of the mice died within the 20-week period. On the other hand, when GcMAF administration was commenced after the 13th week in 5 out of the 15 hamsters in the control group, tumor growth was slightly suppressed and all 15 hamsters died of tumor burden. However, the mean survival time was significantly extended. GcMAF treatment activated peritoneal macrophages in vitro and in vivo, and these activated macrophages exhibited a marked cytocidal effect on HCPC-1 cells. Furthermore, the cytocidal effect of activated macrophages was enhanced by the addition of tumor-bearing hamster serum. These findings
\end{abstract}

Correspondence to: Dr Masahiro Urade, Department of Oral and Maxillofacial Surgery, Hyogo College of Medicine, 1-1 Mukogawa-cho, Nishinomiya, Hyogo 663-8501, Japan

E-mail: uradem@hyo-med.ac.jp

Key words: vitamin D-binding protein-derived macrophageactivating factor, macrophage, 9,10-dimethyl-1,2-benzanthracene, hamster cheek pouch carcinogenesis indicated that GcMAF possesses an inhibitory effect on tumor development and growth in a DMBA-induced hamster cheek pouch carcinogenesis model.

\section{Introduction}

Oral cancer is one of the ten most common cancers in the world, accounting for approximately $2 \%$ of all cancer types and $1 \%$ of all cancer-related deaths (1). Squamous cell carcinoma (SCC) is the most common malignant tumor of the oral cavity, accounting for over $90 \%$ of the malignant neoplasms in this region (2). Despite recent advances in the diagnosis and treatment modalities of surgery, radiotherapy and chemotherapy for oral cancer, the 5-year survival rate has improved only marginally (2). This result indicates the limitations of these treatment modalities. Therefore, additional treatment strategies, such as immunotherapy, are required to improve the 5 -year survival rate.

Cancer patients often suffer from immunodeficiency and increased susceptibility to infection, resulting in death (3). Macrophage activation in phagocytosis and subsequent antigen presentation are involved in immune development, and the capacity of macrophages to be activated is indicative of host immune potential (4). Serum vitamin D3-binding protein (Gc protein) is the precursor for the principal macrophage activating factor (MAF). The MAF precursor activity of the serum Gc protein of various cancer patients, including oral cancer patients, was lost or reduced since the Gc protein is deglycosylated by serum $\alpha$-N-acetyl galactosaminidase (Nagalase) secreted from cancer cells (5). Deglycosylated Gc protein cannot be converted to MAF, leading to immunosuppression. Administration of the Gc protein-derived MAF (GcMAF) that was generated enzymatically in vitro from the Gc protein in human serum, bypasses the impaired macrophage activation cascade and efficiently activates macrophages. Highly activated macrophages have been reported to have a tumoricidal potential (6-9). Pilot studies have reported the efficacy of GcMAF-based immunotherapy of metastatic cancer in animal and humans (10-13).

The aim of the present study was to investigate whether or not GcMAF has an inhibitory effect on oral carcinogenesis 
Schedule of the experiment

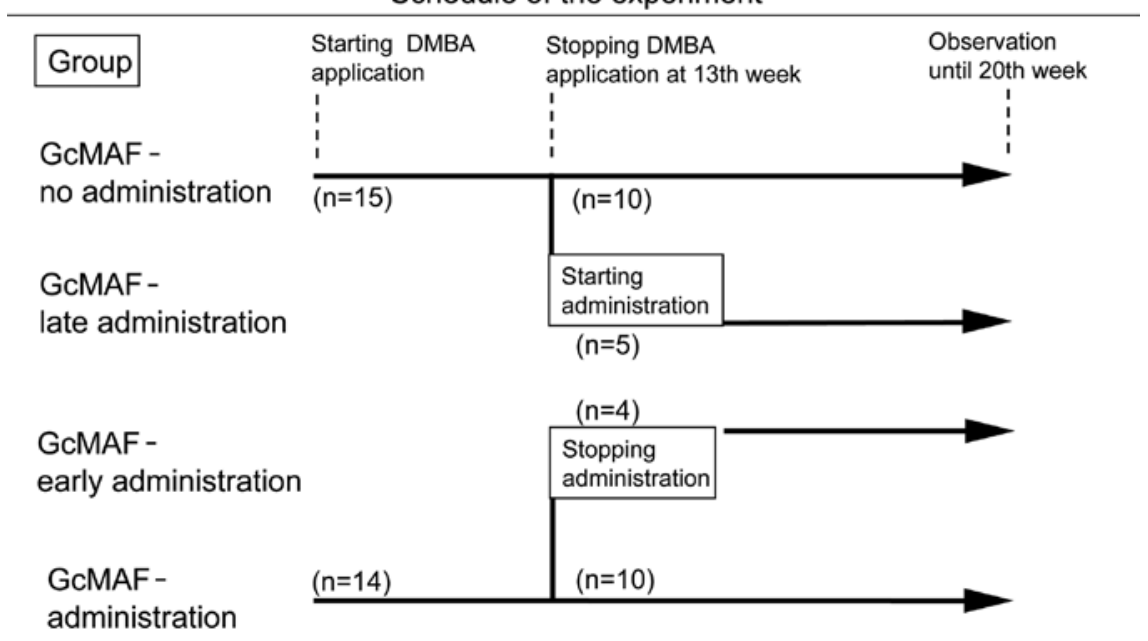

Figure 1. Schedule of the experiment. A total of 14 hamsters were intramuscularly injected with $100 \mathrm{pg} / \mathrm{hamster}$ of GcMAF twice a week from the time period that DMBA application commenced in cheek pouches, while 15 hamsters without GcMAF administration served as controls. From the 13th week, when tumors had formed in all hamsters, GcMAF administration was started in 5 out of the 15 hamsters in the control group (late administration of GcMAF) and stopped in 4 out of the 14 hamsters in the GcMAF-treated group (early administration of GcMAF). Tumor diameter and body weight of hamsters were measured once a week.

and tumor growth, using a 9,10-dimethyl-1,2-benzanthracene (DMBA)-induced hamster cheek pouch carcinogenesis model. The cytocidal effect of GcMAF on its derived squamous carcinoma cell line, HCPC-1, was also been examined, as well as the possible combination of immunotherapy with GcMAF for oral cancer.

\section{Materials and methods}

Preparation of GcMAF. Human serum was heat-inactivated at $60^{\circ} \mathrm{C}$ for $1 \mathrm{~h}$ and $\mathrm{Gc}$ protein fraction was precipitated by mixing with $30 \%$ saturated ammonium (14).

The precipitate was dissolved in phosphate-buffered saline (PBS) ( $\mathrm{pH} 7.4)$ containing $0.5 \%$ Triton X-100 and $0.3 \%$ tri-n-butyl phosphate, and was maintained overnight at room temperature to resolve the lipid containing microbial contaminants. The sample was precipitated by $30 \%$ saturated ammonium sulfate, dissolved in $50 \mathrm{mM}$ citrate buffer at $\mathrm{pH} 4.0$ and maintained overnight. Gc protein was purified using 25-hydroxyvitamin D3-affinity chromatography (15). Stepwise digestion of purified Gc protein with immobilized $\beta$-galactosidase and sialidase yielded the most potent macrophage activating factor (GcMAF) $(16,17)$.

The immobilized enzymes were removed by centrifugation. The final product, GcMAF, was filtered through a low proteinbinding filter, Millex-HV (Millipore Corp., Bedford, MA, USA) for sterilization.

Animals, carcinogen treatment and GcMAF administration. A total of 29 male golden Syrian hamsters, at 5 weeks of age, were purchased from Nihon Animal Inc. (Osaka, Japan). The animals were divided into two groups: 14 hamsters with GcMAF administration by intramuscular injection with $100 \mathrm{pg} /$ hamster to the thigh twice a week from the beginning of DMBA application, while 15 hamsters without GcMAF administration served as controls (Fig. 1). The GcMAF dose of $100 \mathrm{pg} / \mathrm{hamster}$ was employed according to the study reported previously in mice bearing Ehlich ascites tumor (10). These hamsters were treated under ether anesthesia by painting a cheek pouch three times a week with $1 \%$ solution of DMBA (Wako Pure Chemical Industries, Ltd., Osaka, Japan) dissolved in acetone, as previously described $(18,19)$. DMBA application was continued until the 13th week. The diameter of tumors formed was measured using calipers and the body weight of the hamsters was simultaneously measured once a week.

From the 13th week, when tumors had formed on the cheek pouches of all of the hamsters, GcMAF administration was started by intramuscular injection with $100 \mathrm{pg} / \mathrm{hamster}$ twice a week in 5 out of the 15 hamsters in the control group (late administration of GcMAF) and stopped in 4 out of the 14 hamsters in the GcMAF-treated group (early administration of GcMAF).

Animal experiments were performed in compliance with the Guidelines for Animal Experiments of the Hyogo College of Medicine.

Superoxide generation assay. Peritoneal cells were collected by peritoneal lavage with cold PBS, washed three times in cold PBS and plated in a 16-mm multiwell plate in DMEM (Gibco-BRL, Grand Island, NY, USA) supplemented with $1 \%$ fetal bovine serum (FBS; Hyclone Laboratories, South Logan, UT, USA). The cells were incubated at $37^{\circ} \mathrm{C}$ for $30 \mathrm{~min}$ to facilitate the adherence of macrophages to plastic substrate and then washed with PBS gently to remove non-adherent cells. Various concentrations of GcMAF were added and the cells were incubated at $37^{\circ} \mathrm{C}$ for $3 \mathrm{~h}$. To measure the activation of macrophages, the culture medium was replaced with $1 \mathrm{ml}$ of PBS containing $20 \mu \mathrm{g}$ of cytochrome C (Sigma-Aldrich Co., St. Louis, MO, USA) and incubated for $10 \mathrm{~min}$. Approximately $30 \mathrm{~min}$ after the addition of $10 \mu \mathrm{l}$ PBS containing $0.5 \mu \mathrm{g}$ of phorbol 12-myristyl acetate (PMA; Sigma-Aldrich Co.), the superoxide-generating activity of macrophages was determined by measuring the absorbance at $550 \mathrm{~nm}$. 
Table I. Effect of GcMAF administration on DMBA-induced hamster cheek pouch carcinogenesis.

\begin{tabular}{|c|c|c|c|c|c|c|c|}
\hline \multirow[t]{2}{*}{ Treatment } & \multirow[t]{2}{*}{$\begin{array}{c}\text { Tumor } \\
\text { prevalence }(\%)\end{array}$} & \multirow{2}{*}{$\begin{array}{l}\text { Onset of tumor } \\
\text { formation } \\
\text { (weeks) }\end{array}$} & \multicolumn{3}{|c|}{$\begin{array}{l}\text { Tumor diameter } \\
(\mathrm{mm})\end{array}$} & \multirow[t]{2}{*}{$\begin{array}{c}\text { Tumor } \\
\text { death }(\%)\end{array}$} & \multirow[t]{2}{*}{$\begin{array}{l}\text { Mean survival } \\
\text { time (weeks) }\end{array}$} \\
\hline & & & 13th week & 16th week & Ratio & & \\
\hline $\begin{array}{l}\text { GcMAF-no } \\
\text { administration }(n=10)\end{array}$ & $10 / 10(100)$ & $9.9 \pm 0.9$ & $5.3 \pm 3.9$ & $17.9 \pm 5.0$ & 3.38 & $10 / 10(100)$ & $15.0 \pm 2.1$ \\
\hline $\begin{array}{l}\text { GcMAF-late } \\
\text { administration }(n=5)\end{array}$ & $5 / 5(100)$ & & $3.0 \pm 1.5$ & $8.0 \pm 2.9$ & 2.67 & $5 / 5(100)$ & $17.4 \pm 0.5^{b}$ \\
\hline $\begin{array}{l}\text { GcMAF-early } \\
\text { administration }(n=4)\end{array}$ & $4 / 4(100)$ & $13.4 \pm 0.8$ & $2.3 \pm 1.8$ & $7.7 \pm 2.7$ & 3.35 & $0 / 4(0)$ & $>20^{c}$ \\
\hline $\begin{array}{l}\text { GcMAF- } \\
\text { administration }(n=10)\end{array}$ & 8/10 (80) & & $1.5 \pm 1.2$ & $2.8 \pm 2.5$ & $1.87^{\mathrm{a}}$ & 0/8 (0) & $>20^{c}$ \\
\hline
\end{tabular}

Data are the average $\pm \mathrm{SD} .{ }^{\mathrm{a}} \mathrm{p}<0.05,{ }^{\mathrm{b}} \mathrm{p}<0.01,{ }^{\mathrm{c}} \mathrm{p}<0.005$.

For the in vivo activation assay of macrophages, GcMAF (100 pg/hamster) was injected into the thigh of hamsters intramuscularly. The peritoneal cells were harvested 48 to $96 \mathrm{~h}$ after injection and assayed for superoxide generation as described above.

Culture of hamster HCPC-1 cells and treatment with GcMAF-activated macrophages. HCPC-1 cells were isolated and established from the 7,12-dimethylbenz $(\alpha)$ anthraceneinduced epidermoid carcinoma of golden Syrian hamster cheek pouch. The cells were kindly provided by Dr G. Shklar (Harvard School of Dental Medicine, Boston, MA, USA) (20). To examine the cytocidal effect of GcMAF-activated macrophages on $\mathrm{HCPC}-1$ cells, the cells were plated at a density of $10^{5}$ cells/well in DMEM supplemented with $10 \%$ FBS in a multiwell plate and incubated at $37^{\circ} \mathrm{C}$ for $24 \mathrm{~h}$. Non-activated macrophages or macrophages activated with GcMAF in vitro or in vivo were then added at the effector to target ratio (E:T ratio) of 5:1 and further incubated at $37^{\circ} \mathrm{C}$ for $48 \mathrm{~h}$. Peritoneal macrophages were activated either by intramuscular injections with $100 \mathrm{pg}$ GcMAF twice (1 and 4 days prior to collection) in vivo or by the addition of $100 \mathrm{pg}$ GcMAF to the medium and incubation at $37^{\circ} \mathrm{C}$ for $1 \mathrm{~h}$ in vitro. Viable HCPC-1 cells were counted at constant intervals by the nigrosin exclusion test in a hemocytometer.

The effect of the addition of heat-inactivated DMBAinduced tumor-bearing hamster serum on macrophagedirected cytotoxicity with GcMAF in HCPC-1 cells was also studied, since it was reported that the tumoricidal activity of activated macrophages was markedly enhanced by the addition of tumor-bearing patient serum in the human retinoblastoma cell line W24 (6).

The tumor-bearing hamster serum, 20 weeks after DMBA application, was collected from 5 hamsters and mixed together just before the experiment. The tumor-bearing or normal hamster serum was added to the culture medium at the final concentration of $5 \%$ after heat-inactivation at $56^{\circ} \mathrm{C}$ for $30 \mathrm{~min}$.
Statistical analysis. Statistical analysis of the data was performed by using the Student's t-test. $\mathrm{P}<0.05$ was considered to be statistically significant.

\section{Results}

Suppression of carcinogenesis and tumor growth by GcMAF administration. SCC was produced from the 9th to the 11th week after DMBA application in all 15 hamsters of the control group without GcMAF administration, and all died of tumor burden within 20 weeks. Out of the 14 hamsters, 2 animals with GcMAF administration did not develop tumors, while the remaining 12 hamsters showed a significant delay of tumor development for $\sim 3.5$ weeks in addition to suppressed tumor growth. These 12 hamsters survived until the 20th week of the experimental period (Table I and Fig. 2). The body weight loss associated with tumor burden was significantly higher in the control group than that in the GcMAF-treated group (Fig. 3).

When GcMAF administration was started from the 13th to the 20th week in 5 tumor-bearing hamsters in the control group, tumor growth was slightly suppressed and all of the hamsters died of tumor burden; however, body weight loss was significantly inhibited and the mean survival time was extended. In contrast, when GcMAF administration was stopped in the 13th week in 4 tumor-bearing hamsters in the GcMAF-treated group, tumor growth and body weight loss were promoted, but none of the hamsters died within the 20-week period (Table I, Figs. 2 and 3).

Macrophage activation with GcMAF assayed by superoxide generation. When hamster peritoneal macrophages were treated with various concentrations of GcMAF in vitro, superoxide generation was increased 9-fold in a dose-dependent manner, indicating that the macrophage activation needed to cause efficient tumoricidal effect (Table II). Intramuscular administration of GcMAF to hamsters also showed an $\sim 3$-fold increase of superoxide generation in macrophages as compared to the control at 48 and $96 \mathrm{~h}$ post injection. 


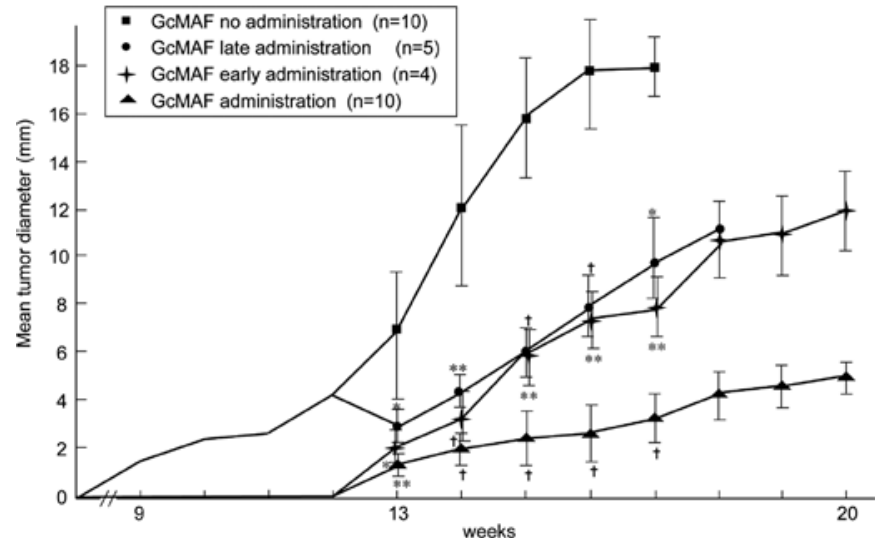

Figure 2. Tumor growth curves in treatment with or without GcMAF. Hamsters were divided into two groups with or without GcMAF until the 13th week and then subdivided into four groups as shown in Fig. 1. Tumor diameters were measured until the 20th week. $\mathbf{m}$, no administration of GcMAF $(\mathrm{n}=10)$; $\bullet$, late administration of GcMAF $(\mathrm{n}=5)$; $\bullet$, early administration of $\operatorname{GcMAF}(n=4) ; \mathbf{\Delta}$, administration of GcMAF $(n=10)$. Data are the average \pm SD. ${ }^{*} \mathrm{p}<0.05 ;{ }^{* *} \mathrm{p}<0.01 ;{ }^{\dagger} \mathrm{p}<0.005$

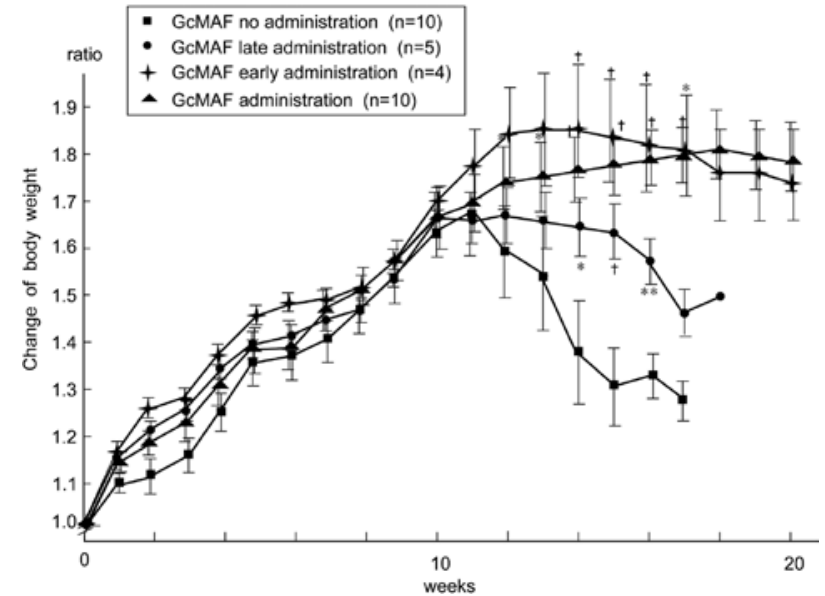

Figure 3. Changes of the body weight of tumor-bearing hamsters with or without GcMAF administration. The body weight of hamsters was measured once a week in each group. $\mathbf{m}$, no administration of GcMAF (n=10); $\bullet$, late administration of GcMAF ( $n=5)$; , early administration of GcMAF $(n=4)$; $\Delta$, administration of GcMAF $(n=10)$. Data are the average \pm SD. " $p<0.05$; ${ }^{*} \mathrm{p}<0.01 ;{ }^{\dagger} \mathrm{p}<0.005$

Table II. Superoxide generation in macrophages with GcMAF treatment in vitro and in vivo.

\section{GcMAF treatment}

Generation of superoxide $\left(\mathrm{nmol} / \mathrm{min} / 10^{6}\right.$ cells $)$

\begin{tabular}{lll}
\hline In vitro experiment (treatment with GcMAF at $37^{\circ} \mathrm{C}$ for $\left.3 \mathrm{~h}\right)$ & & 1.0 \\
GcMAF no administration & $0.094 \pm 0.02$ & 2.2 \\
$\mathrm{GcMAF}(0.1 \mathrm{pg} / \mathrm{ml})$ & $0.209 \pm 0.04$ & 3.6 \\
$\mathrm{GcMAF}(1 \mathrm{pg} / \mathrm{ml})$ & $0.335 \pm 0.04$ & 6.2 \\
$\mathrm{GcMAF}(10 \mathrm{pg} / \mathrm{ml})$ & $0.586 \pm 0.05$ & 9.0 \\
$\mathrm{GcMAF}(100 \mathrm{pg} / \mathrm{ml})$ & $0.848 \pm 0.06$ & 1.0 \\
In vivo experiment (intramuscular injection of $100 \mathrm{pg}$ of GcMAF/hamster) & & 3.1 \\
GcMAF no administration & $0.094 \pm 0.02$ & 3.4 \\
$48 \mathrm{~h}$ post injection & $0.293 \pm 0.03$ & $0.324 \pm 0.05$ \\
$96 \mathrm{~h}$ post injection & & \\
\hline
\end{tabular}

Peritoneal macrophages were treated with various concentrations of GcMAF in vitro at $37^{\circ} \mathrm{C}$ for $3 \mathrm{~h}$ and superoxide generated was assayed as described in Materials and methods. In the in vivo experiment, GcMAF 100 pg/hamster was intramuscularly injected into the thigh of each hamster and superoxide generation was assayed for peritoneal macrophages harvested 48 and $96 \mathrm{~h}$ after injection $(\mathrm{n}=6)$.

Table III. Cytocidal effect of peritoneal macrophages activated with GcMAF in vitro on HCPC-1 cells.

\begin{tabular}{lcr}
\hline Macrophages $(\mathrm{M} \phi)$ treated & No. of viable HCPC-1 cells $\left(\mathrm{x} 10^{4}\right)$ & Ratio \\
\hline Control & $100.3 \pm 10.0$ & 1.00 \\
Non-activated M $\phi$ & $69.0 \pm 4.5$ & $0.69^{\mathrm{a}}$ \\
Activated M $\phi$ with 1 pg of GcMAF & $66.5 \pm 3.4$ & $0.66^{\mathrm{a}}$ \\
Activated M $\phi$ with 10 pg of GcMAF & $62.3 \pm 4.6$ & $0.62^{\mathrm{a}}$ \\
Activated M $\phi$ with 100 pg of GcMAF & $54.8 \pm 5.6$ & $0.55^{\text {a }}$ \\
Activated M $\phi$ with 200 pg of GcMAF & $54.3 \pm 2.6$ & $0.54^{\mathrm{a}}$ \\
\hline
\end{tabular}

The non-activated macrophages $(\mathrm{M} \phi)$ or macrophages activated with GcMAF were added to the HCPC-1 culture at an E:T ratio of 5:1 and further incubated at $37^{\circ} \mathrm{C}$ for $48 \mathrm{~h}$. Peritoneal macrophages were activated by the addition of various concentrations of GcMAF to the medium and incubated at $37^{\circ} \mathrm{C}$ for $1 \mathrm{~h}$ in vitro. Viable HCPC-1 cells were counted by the nigrosin exclusion test in a hemocytometer. Data are the average $\pm \operatorname{SD}(n=4) .{ }^{\mathrm{a}} \mathrm{p}<0.005$. 
Table IV. Comparison of cytocidal effect of peritoneal macrophages activated with GcMAF in vitro and in vivo on HCPC-1 cells.

\begin{tabular}{lcr}
\hline Macrophages $(\mathrm{M} \phi)$ treated & No. of viable HCPC-1 cells $\left(\times 10^{4}\right)$ & Ratio \\
\hline Control & $93.6 \pm 9.0$ & 0.90 \\
GcMAF & $83.6 \pm 11.0$ & 0.76 \\
Non-activated $\mathrm{M} \phi$ & $71.6 \pm 1.8$ & $0.66^{\mathrm{a}}$ \\
In vitro activated $\mathrm{M} \phi$ & $61.9 \pm 3.0$ & $0.42^{\mathrm{b}}$ \\
In vivo activated $\mathrm{M} \phi$ & $39.5 \pm 4.2$ & \\
\hline
\end{tabular}

The HCPC-1 cells were treated with non-activated or activated macrophages with GcMAF in vitro and in vivo as described in Table III. Peritoneal macrophages were activated by the addition of $100 \mathrm{pg}$ of GcMAF at $37^{\circ} \mathrm{C}$ for $1 \mathrm{~h}$ in vitro, or by intramuscular injection with $100 \mathrm{pg}$ of GcMAF twice ( 1 and 4 days prior to collection) in vivo. Viable HCPC-1 cells were counted. ${ }^{\mathrm{a}} \mathrm{p}<0.01,{ }^{\mathrm{b}} \mathrm{p}<0.005$.

Table V. Elevated cytocidal effect of peritoneal macrophages activated with GcMAF in vivo by the addition of tumor-bearing hamster serum on HCPC-1 cells.

\begin{tabular}{lcr}
\hline Macrophages $(\mathrm{M} \phi)$ and serum & No. of viable HCPC-1 cells $\left(\mathrm{x} 10^{4}\right)$ & Ratio \\
\hline Control & $116.4 \pm 15.7$ & 1.00 \\
+ Normal serum & $103.4 \pm 6.7$ & 0.89 \\
+ Tumor-bearing serum & $68.6 \pm 14.1$ & $0.59^{\mathrm{a}}$ \\
+ Non-activated M $\phi$ & $72.0 \pm 4.5$ & $0.62^{\mathrm{b}}$ \\
+ Non-activated $M \phi+$ normal serum & $68.0 \pm 3.8$ & $0.58^{\mathrm{c}}$ \\
+ Non-activated $M \phi+$ tumor-bearing serum & $52.0 \pm 9.6$ & $0.45^{\mathrm{c}}$ \\
+ In vivo activated $\mathrm{M} \phi$ & $53.6 \pm 4.3$ & $0.46^{\mathrm{c}}$ \\
+ In vivo activated $\mathrm{M} \phi+$ normal serum & $50.6 \pm 6.9$ & $0.43^{\mathrm{c}}$ \\
+ In vivo activated $\mathrm{M} \phi+$ tumor-bearing serum & $13.0 \pm 2.6$ & $0.11^{\mathrm{c}}$ \\
\hline
\end{tabular}

The HCPC-1 cells were treated with non-activated or GcMAF-activated macrophages in the presence or absence of tumor-bearing hamster serum at $37^{\circ} \mathrm{C}$ for $48 \mathrm{~h}$. The tumor-bearing hamster or normal serum was added to the culture medium at the final concentration of $5 \%$. Viable HCPC-1 cells were counted. Data are the average $\pm \operatorname{SD}(n=5) .{ }^{a} \mathrm{p}<0.05,{ }^{b} \mathrm{p}<0.01,{ }^{\mathrm{c}} \mathrm{p}<0.005$.

Cytocidal effect of GcMAF-activated macrophages on HCPC-1 cells. The cytocidal effect of GcMAF-activated macrophages was examined by treatment with an E:T ratio of 5:1 at $37^{\circ} \mathrm{C}$ for $48 \mathrm{~h}$ on HCPC-1 cells. As shown in Table III, the number of viable cells was decreased by $69 \%$ even with the addition of non-activated macrophages, but decreased to $\sim 55 \%$ in the case of macrophages activated with 100 and $200 \mathrm{pg}$ of GcMAF. A similar or stronger effect was obtained by treatment with macrophages activated in vivo as well as in vitro (Table IV). To investigate the effect of tumor-bearing hamster serum on macrophage-directed cytotoxicity in HCPC-1 cells, tumor-bearing hamster serum was added at a final concentration of $5 \%$ to the medium. As shown in Table V, the tumor-bearing hamster serum was more cytotoxic than normal serum, and the number of viable cells was decreased by $60 \%$. This was a similar level to that obtained through the addition of non-activated macrophages. However, treatment with GcMAF-activated macrophages reduced the viable cells by $46 \%$ and the combined treatment with GcMAF-activated macrophages and tumor-bearing hamster serum markedly reduced the viable cells by $11 \%$. HCPC-1 cells attacked by macrophages became round and were eradicated (Fig. 4).

\section{Discussion}

Inflammation of cancer tissues induced by the intratumoral administration of Bacillus Calmette-Guerin (BCG) or other bacterial cells has been widely established to result in the regression of local as well as metastasized tumors, suggesting the development of specific immunity against the tumor (21,22). Since cancer tissues comprise alkylphospholipids, inflamed cancer tissues release lysoalkylphospholipids and alkylglycerols (23-26). Lysoalkylphospholipids and alkylglycerols are both potent macrophage-activating agents, and inflammation-derived macrophage activation is the principal macrophage activation process requiring serum vitamin D3-binding protein (Gc Protein) (16,27-31). Hydrolysis of the Gc protein with the inducible membrane $\beta$-galactosidase of inflammation-primed B cells as well as the membranous Neu-1 sialidase of T cells generates GcMAF. GcMAF can be generated enzymatically in vitro by the stepwise treatment of highly purified Gc protein with immobilized $\beta$-galactosidase and sialidase $(10,17,28)$. Pilot studies have reported GcMAF antitumor effects on a variety of cancers, such as colorectal, breast and prostate cancer (11-13). Moreover, $83 \%$ of patients 

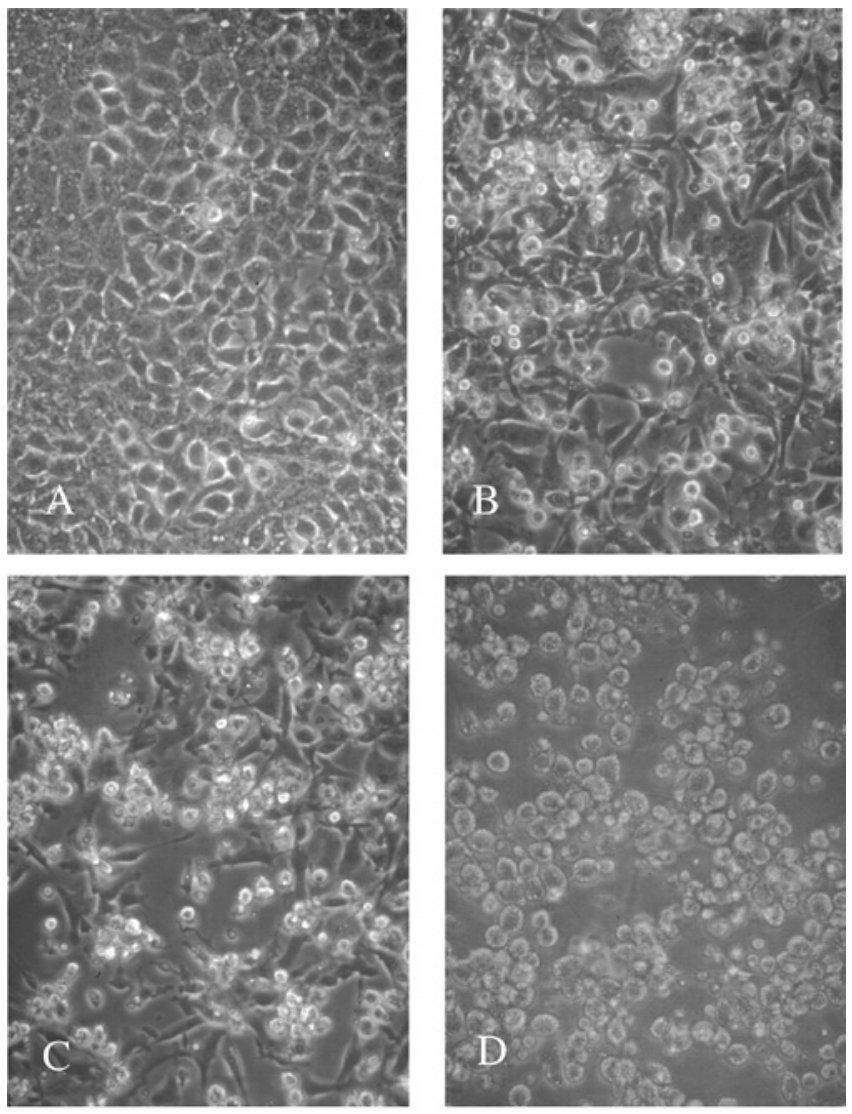

Figure 4. Phase-contrast photomicrographs of HCPC-1 cells following treatment with GcMAF-activated macrophages and tumor-bearing hamster serum The HCPC- 1 cells were incubated at $37^{\circ} \mathrm{C}$ for $24 \mathrm{~h}$ and then non-activated macrophages or macrophages activated with GcMAF in vivo were added at an E:T ratio of 5:1 and further incubated at $37^{\circ} \mathrm{C}$ for 48 h. (A) Control, (B) nonactivated macrophages and tumor-bearing hamster serum, $(\mathrm{C})$ in vivo activated macrophages and normal hamster serum, and (D) in vivo activated macrophages and tumor-bearing hamster serum. Original magnification, x200.

with oral SCC were found to have decreased precursor activity of serum Gc protein and, by contrast, increased Nagalase activity, which efficiently deglycosylated Gc protein (5). Therefore, exogenous administration of GcMAF is considered to inhibit oral carcinogenesis and tumor growth. This study aimed to investigate the antitumor effect of GcMAF on oral cancer by using a well-established DMBA-induced hamster cheek pouch carcinogenesis model and its derived carcinoma cell line.

Consequently, administration of GcMAF from the beginning of DMBA application to cheek pouches resulted in decreased tumor prevalence and a significant delay in tumor formation. Tumor growth was retarded significantly and no death due to tumor burden was noted until the 20th week of observation.

On the other hand, all hamsters without GcMAF administration developed tumors by the 11th week and died by the 20th week. This antitumor effect of GcMAF was further evidenced by starting or stopping GcMAF administration halfway through the experiment. On the other hand, tumor growth was slightly suppressed when GcMAF administration was commenced from the halfway point, but the mean survival time was prolonged significantly. On the other hand, stopping GcMAF administration at the halfway point promoted tumor growth, but maintained no tumor death and the mean survival time was extended (Fig. 2 and Table I). In this case, since the mean tumor diameter between two subgroups of 10 hamsters without GcMAF administration and 5 hamsters with late administration of GcMAF differed by more than 2-fold, the results in tumor growth and mean survival time may be biased. However, when the two subgroups of early and late administration of GcMAF were compared, early administration of GcMAF was found to be more effective in the elongation of life span than late administration. In addition, GcMAF administration has no observed side effects and has prevented body weight loss in tumor-bearing animals (Fig. 3). To evaluate the macrophage activation with GcMAF, the generation of superoxide from peritoneal macrophages was measured. Superoxide was efficiently generated in a dose-dependent manner by in vitro treatment with GcMAF. Similarly, in vivo treatment with GcMAF generated superoxide to the same extent at 48 and $96 \mathrm{~h}$ post injection (Table II).

Highly activated macrophages with GcMAF have already been reported to have a strong tumoricidal activity (6-9). In order to investigate the antitumor effect of GcMAF on DMBAinduced hamster cheek pouch carcinogenesis, tumoricidal activity of GcMAF-activated macrophages was examined using HCPC-1, which is a squamous carcinoma cell line derived from DMBA-induced cheek pouch carcinoma. Consequently, macrophages activated in vitro and in vivo with GcMAF demonstrated a significant tumoricidal activity on HCPC-1 cells, as compared to non-activated macrophages. This activity was dosedependent with a plateau in 100 pg administration of GcMAF (Tables III and IV). Since it was reported that the tumoricidal activity of macrophages photodynamically activated with hematoporphyrin derivative was markedly enhanced on human retinoblastoma cells by the addition of retinoblastoma patient serum in culture (6), the effect of the addition of tumor-bearing hamster serum was tested on tumoricidal activity for HCPC-1 cells. As expected, a marked tumoricidal activity for HCPC-1 cells was observed and $89 \%$ of the cells were killed in contrast to $57 \%$ of cells killed by activated macrophages and normal hamster serum (Table V). This finding suggests that tumorbearing hamsters carry IgG-antibodies against antigens, such as tumor-associated antigen (32-35) for cheek pouch carcinoma. Additionally, Fc-receptors of activated macrophages promote the ingestion of tumor cells. Since GcMAF-treated macrophages develop a large number of Fc-receptors $(4,17,36)$, this enhanced cell killing is considered to be due to an Fc-receptormediated process.

Although GcMAF therapy has been found to show curative effects on a variety of cancers $(10-13,37,38)$, Yamamoto et al stated that the efficacy of GcMAF therapy for a variety of cancer types depends on the degree of cell membrane abnormality $(12,13)$. Undifferentiated tumor cells are killed more efficiently than differentiated tumor cells. Adenocarcinoma, such as breast and prostate cancer, is undifferentiated and killed rapidly by the activated macrophages, whereas welldifferentiated tumor cells, such as SCC cells, are slowly killed by the activated macrophages. SCC occupies approximately $90 \%$ of oral cancer, the majority of which is well-differentiated SCC. DMBA-induced cheek pouch carcinoma is also welldifferentiated SCC. These facts suggest that GcMAF therapy is not as efficacious in oral cancer as compared to other types 
of cancer. In the present study, however, GcMAF exhibited a marked retardation in tumor development and growth was demonstrated by, along with increased survival time, without any noteworthy side effects. GcMAF treatment may therefore have therapeutic potential for oral cancer, as supported by this animal model.

\section{Acknowledgements}

This study was supported by a Grant-in-Aid for Scientific Research from the Ministry of Education, Science and Culture of Japan (no. 09672086 to M.U. and no. 11771304 to H.K.), and a Grant-in-Aid for Researchers, Hyogo College of Medicine, to S.H.

\section{References}

1. Parkin DM, Bray F, Ferlay J and Pisani P: Global cancer statistics, 2002. CA Cancer J Clin 55: 74-108, 2005.

2. Von Dongen GA and Snow GB: Prospectives for future studies in head and neck cancer. Eur J Surg Oncol 23: 485-491, 1997.

3. Gross L: Immunological defect in aged population and its relationship to cancer. Cancer Res 18: 201-204, 1965.

4. Ngwenya BZ and Yamamoto N: Activation of peritoneal macrophages by lysophosphatidylcholine. Biochim Biophys Acta 839: 9-15, 1985

5. Yamamoto N, Naraparaju VR and Urade M: Prognostic utility of serum $\alpha-\mathrm{N}$-acetylgalactosaminidase and immunosuppression resulted from deglycosylation of serum Gc protein in oral cancer patients. Cancer Res 57: 295-299, 1997.

6. Yamamoto $\mathrm{N}$ and Hoober JK: Tumoricidal capacities of macrophages photodynamically activated with hematoporphyrin derivative. Photochem Photobiol 56: 245-250, 1992.

7. Fidler JJ and Schroit AJ: Recognition and destruction of neoplastic cells by activated macrophages: discrimination of altered self. Biochim Biophys Acta 948: 151-173, 1988.

8. Keller R: The macrophage response to infectious agents: mechanisms of macrophage activation and tumor cell killing. Res Immunol 144: 271-273, 1993.

9. Klostergard J: Macrophage tumoricidal mechanisms. Res Immunol 144: 274-276, 1993.

10. Yamamoto $\mathrm{N}$ and Naraparaju VR: Immunotherapy of BALB/c mice bearing Ehrlich ascites tumor with vitamin D-binding protein-derived macrophage activating factor. Cancer Res 57: 2187-2192, 1997.

11. Yamamoto N, Suyama H, Nakazato H, Yamamoto NY and Koga Y: Immunotherapy of metastatic colorectal cancer with vitamin D-binding protein-derived macrophage activating factor, GcMAF. Cancer Immunol Immunother 57: 1007-1016, 2007.

12. Yamamoto N, Suyama H, Yamamoto NY and Ushijima N: Immunotherapy of metastatic breast cancer patients with vitamin D-binding protein-derived macrophage activating factor (GcMAF). Int J Cancer 122: 461-467, 2008.

13. Yamamoto N, Suyama H and Yamamoto N: Immunotherapy for prostate cancer with $\mathrm{Gc}$ protein-derived macrophage-activating factor, GcMAF. Transl Oncol 1: 65-72, 2008

14. Yamamoto N, Willett NP and Lindsay DD: Participation of serum proteins in the inflammation-primed activation of macrophages. Inflammation 18: 311-322, 1994.

15. Link RP, Perlman KL, Pierce EA, Schnoes HK and DeLuca HF: Purification of human serum vitamin D-binding protein by 25-hydroxyvitamin D3-Sepharose chromatography. Anal Biochem 157: 262-269, 1986.

16. Yamamoto $\mathrm{N}$ and Kumashiro R: Conversion of vitamin D3 binding protein (group-specific component) to a macrophage activating factor by the stepwise action of beta-galactosidase of $\mathrm{B}$ cells and sialidase of T cells. J Immunol 151: 2794-2802, 1993.

17. Yamamoto N: Structural definition of a potent macrophage activating factor derived from vitamin D3-binding protein with adjuvant activity for antibody production. Mol Immunol 33: $1157-1164,1996$

18. Nishimura N, Urade $\mathrm{M}$, Hashitani $\mathrm{S}$, et al: Increased expression of cyclooxygenase (COX)-2 protein in DMBA-induced hamster cheek pouch carcinogenesis and chemopreventive effect of a selective COX-2 inhibitor celecoxib. J Oral Pathol Med 33: 614-621, 2004
19. Segawa E, Hashitani S, Toyohara Y, et al: Inhibitory effect of sulindac on DMBA-induced hamster cheek pouch carcinogenesis and its derived cell line. Oncol Rep 21: 869-874, 2009.

20. Odukoya O, Schwartz J, Weichselbaum R and Shklar G: An epidermoid carcinoma cell line derived from hamster 7,12-dimethylbenz $(\alpha)$ anthracene-induced buccal pouch tumors. J Natl Cancer Inst 71: 1253-1264, 1983.

21. Zbar B and Tanaka T: Immunotherapy of cancer: regression of tumors after intralesional injection of living Mycobacterium bovis. Science 172: 271-273, 1971.

22. Morton D, Eibler FR, Malmgren RA and Wood WC: Immunological factors which influence response to immunotherapy in malignant melanoma. Surgery 68: 158-164, 1970.

23. Yamamoto $\mathrm{N}$ and Ngwenya BZ: Activation of mouse peritoneal macrophages by lysophospholipids and ether derivatives of neutral lipids and phospholipids. Cancer Res 47: 2008-2013, 1987.

24. Yamamoto N, Ngwenya BZ and Pieringer PA: Activation of macrophages by ether analogues of lysophospholipids. Cancer Immunol Immunother 25: 185-192, 1987.

25. Yamamoto N, St Claire DA Jr, Homma S and Ngwenya BZ: Activation of mouse macrophages by alkylglycerols, inflammation products of cancerous tissues. Cancer Res 48: 6044-6049, 1988.

26. Homma S and Yamamoto N: Activation process of macrophage after in vitro treatment of mouse lymphocytes with dodecylglycerol. Clin Exp Immunol 79: 307-313, 1990.

27. Homma S, Yamamoto M and Yamamoto N: Vitamin D binding protein (group-specific component, Gc) is the sole serum protein required for macrophage activation after treatment of peritoneal cells with lysophosphatidylcholine. Immunol Cell Biol 71: 249-257, 1993.

28. Naraparaju VR and Yamamoto N: Roles of beta-galactosidase of B lymphocytes and sialidase of T lymphocytes in inflammationprimed activation of macrophages. Immunol Lett 43: 143-148, 1994.

29. Yamamoto $\mathrm{N}$ and Homma S: Vitamin D3 binding protein (group-specific component) is a precursor for the macrophageactivating signal factor from lysophosphatidylcholine-treated lymphocytes. Proc Natl Acad Sci USA 88: 8539-8543, 1991.

30. Yamamoto N, Homma S, Haddad JG and Kowalski MN: Vitamin D3 binding protein required for in vitro activation of macrophages after dodecylglycerol treatment of mouse peritoneal cells. Immunology 74: 420-424, 1991.

31. Yamamoto N, Homma S and Millman I: Identification of the serum factor required for in vitro activation of macrophage role of vitamin D3-binding protein (group specific component, $\mathrm{Gc}$ ) in lysophospholipid activation of mouse peritoneal macrophages. J Immunol 147: 273-280, 1991.

32. Zhang S, Cordon-Cardo C, Zhang HS, et al: Selection of tumor antigens as targets for immune attack using immunohistochemistry. I. Focus on gangliosides. Int J Cancer 73: 42-49, 1997.

33. Zhang S, Zhang HS, Cordon-Cardo C, et al: Selection of tumor antigens as targets for immune attack using immunohistochemistry. II. Blood group-related antigens. Int J Cancer 73: 50-56, 1997.

34. Zhang S, Zhang HS, Cordon-Cardo C, Ragupathi G and Livingston PO: Selection of tumor antigens as targets for immune attack using immunohistochemistry. III. Protein antigen. Clin Cancer Res 4: 2669-2676, 1998.

35. Musselli C, Ragupathi G, Gilewski T, Panageas KS, Spinat Y and Livingston PO: Reevaluation of the cellular immune response in breast cancer patients vaccinated with MUC1. Int J Cancer 97: 660-667, 2002.

36. Ngwenya BZ and Yamamoto N: Effects of inflammation products on immune systems: lysophosphatidylcholine stimulates macrophages. Cancer Immunol Immunother 21: 174-182, 1986.

37. Yamamoto N, Urade $\mathrm{M}$ and Ueda $\mathrm{M}$ : Potent tumoricidal capacity of macrophages activated by Gc protein-derived macrophage activating factor (GcMAF) and its therapeutic efficacy for prostate, breast and colorectal cancers. J Immunother 28: 642, 2005.

38. Yamamoto $\mathrm{N}$ and Ueda $\mathrm{M}$ : Therapeutic efficacy of vitamin D-binding protein (Gc protein)-derived macrophage activating factor (GcMAF) for prostate and breast cancers. In: Immunology. Medmond Ltd., Bologna, Italy, pp201-204, 2004. 